\title{
Understanding properties of hydrogen storage materials from local structure using PDF technique
}

\section{OKim Hyunjeong （産業技術総合研究所）}

One of the safe and efficient ways to store a large amount of hydrogen is utilizing hydrogen absorbing materials. For practical applications, however, several material challenges still remain to be tackled. Various new ideas and approaches have been proposed and tested in an effort to improve the hydrogen storage properties of conventional materials. The interesting properties of such novel materials often arise from nanoscale or heavily disordered structural features that are difficult to characterize using conventional crystallographic methods. In order to obtain their structural information, we have been using the atomic pair distribution function (PDF) analysis [1] on total scattering data obtained at Synchrotron X-ray or spallation neutron sources. The PDF is a local structural technique that is widely used for studying various types of materials these days. Using the PDF, we have investigated nano-confined $\mathrm{NH}_{3} \mathrm{BH}_{3}$ [2], mechanically alloyed Mg-Co alloys [3] and heavily disordered vanadium-based alloys [4]. These materials do not show well defined Bragg peaks in their diffraction patterns and it is quite challenging to obtain structural information. In this talk, I will show how their structural information is extracted from the PDFs and how it is used to understand their hydrogen storage properties.

[1] T. Egami and S. J. L. Billinge, Underneath the Bragg Peaks: Structural Analysis of Complex Materials: Pergamon Press Elsevier: Oxford, England, 2003.

[2] H. Kim et al., J. Am. Chem. Soc. 131, 13749-13755 (2009).

[3] H. Kim et al., J. Phys. Chem. C 115, 20335-20341 (2011).

[4] H. Kim et al., J. Phys. Chem. C 117, 26543-26550 (2013).

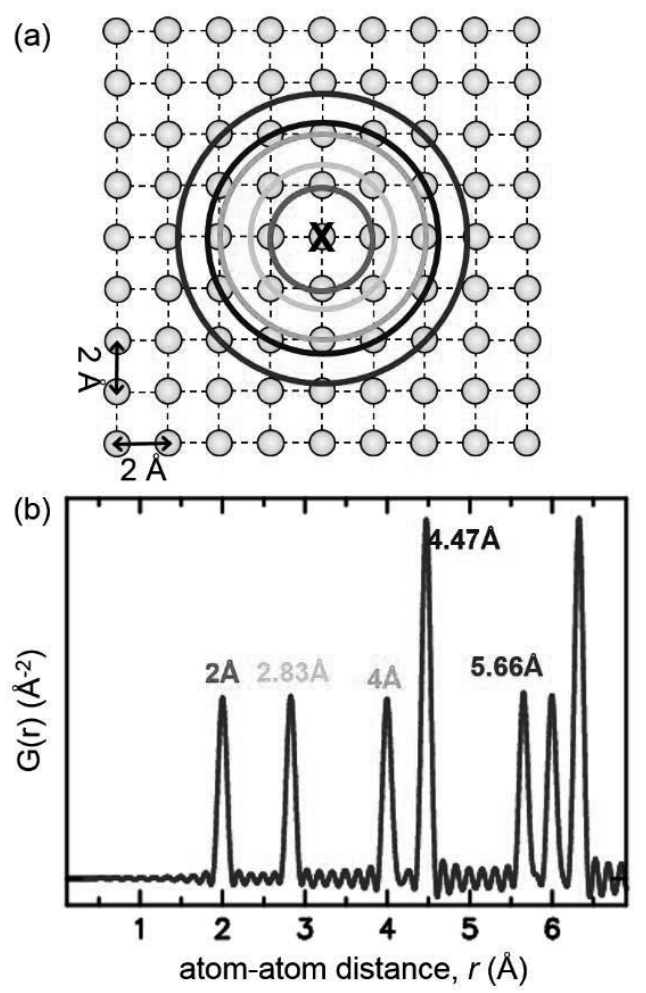

図 1 (a) A two dimensional atomic arrangement and (b) the corresponding PDF. 